moniumbifluorid des Handels der Zusammensetzung $\mathrm{NH}_{4} \mathrm{FFH}$ entspricht, da ich bei meinen früheren Untersuchungen zu dem in den Monatsheften für Chemie (1907, 1148) mitgeteilten Ergebnisse gekommen war, daß ,das Fluorammonium des Handels, welches häufig auch saures Fluorammonium genannt wird, die Zusammensetzung $\mathrm{NH}_{4} \mathrm{~F}$ besitzt und nicht $\mathrm{NH}_{4} \mathrm{FFH}^{\prime \prime}$.

Zur Wertbestimmung des Ammoniumbifluorids wägt man $0,05-0,08 \mathrm{~g}$ des Salzes in einem Platintiegel (Rauminhalt ungefähr $20 \mathrm{ccm}$ ), gibt einige Tropfen Wasser hinzu und dann einen reichlichen therschuß von eingestellter Barytlauge, bedeckt den Tiegel mit einem Uhrglase und läßt ihn unter einer Glasglocke die Nacht über stehen, darauf wird der bedeckte Tiegel auf einem Wasserbade langsam angewärmt und allmählich immer stärker bis zum Sieden des Wasserbades erhitzt, was un- gefähr 4-5 Stunden in Anspruch nimmt. Man entfernt dann das Uhrglas vom Tiegel und beläßt diesen noch einige Minuten auf dem siedenden Wasserbade, bis $\mathrm{NH}_{\mathbf{s}}$-Geruch kaum mehr wahrnehmbar ist. Man entleert den Inhalt des Tiegels in ein passendes Becherglas, spült mit Wasser einmal nach und erhitzt das Becherglas auf 90-100 bis kein $\mathrm{NH}_{3}$-Geruch mehr zu bemerken ist. Nun wird aus dem Tiegel der Rest von Baryt und Bariumcarbonat mit einer gemessenen Menge n. $\mathrm{HCl}$ herausgelöst und mit Wasser quantitativ in das Becherglas gespült. Hierauf wird noch $\mathrm{n}$. $\mathrm{HCl}$ so viel zugefügt, $\mathrm{da} B$ ein tberschuß davon vorhanden ist. Das Becherglas erwärmt man kurze Zeit und titriert den Uberschuß von Salzsäure mit Baryt (als Indicator Phenolphthalein) zurück.

Es folgen aus den zahlreich angestellten Bestimmungen einige Beleganalysen.

I a) $0,0657 \mathrm{~g}$ Substanz verbrauchten 7,86 com Beryt $=65,91 \%$ HF

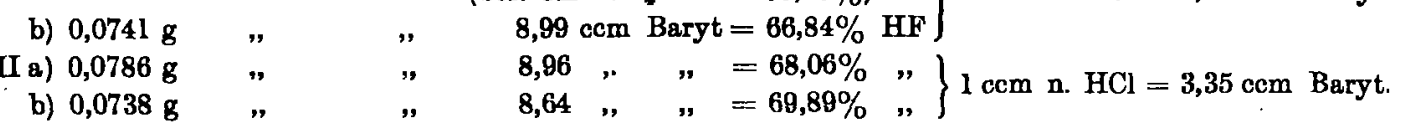

Die Verschiedenheit der einzelnen Werte rührt nicht etwa her von Versuohsfehlern oder von einer Ungenauigkeit der Methode, sondern ist darauf zurückzuführen (wie schon oben angedeutet), daß das in Form gepreßte Ammoniumbifluorid selbst im HF-Gehalte mehr oder minder schwankt. Dies konnte ich mittels der Fluorbestimmungsmethode nach Lenz-DeuB en zur Genüge feststellen.

Ich bin überzeugt, daß die beschriebene Titrationsmethode einwandsfreie und sicherere Werte liefert als die von J. Bra n d empfohlene.

[A. 103:]

\section{Nachweis geringer Mengen Schwefel in anorganischen und organischen Verbindungen.}

\author{
Von Ervst Deussen.
}

(Mitteilung aus dem Institute ron E. Beckmann, Laboratorium für angewandte Chemie der Univ crsitat Leipzig.

(Eingeg. 4./5. 1910.)

In der Zeitschrift f. analyt. Chemie ${ }^{1}$ ) veröffentlichte ich einen qualitativen und quantitativen Nachweis von Schwefelsäure neben Flußsäure, der darauf beruht, daB Sulfat bei Gegenwart von Soda und Filtrierpapier durch die Flammengase einer mit Ligroin gespeisten (sog. schwedischen) Lötrohrlampe in Sulfid übergeführt und dieses durch eine mit Essigsäure angesäuerte Bleiacetatlösung nachgewiesen wird. Die quantitative Bestimmung gestaltet sich - für genquere Zwecke - ein wenig umständlicher, da das Fluorid die Reduktion des Sulfats in Sulfid in der Weise beeinträchtigt, daB weniger Sulfat als tatsächlich im Untersuchungsobjekte vorhanden gefunden wird.

DaB diese Methode sich recht gut zur qualitativen und auch zur raschen, orientierenden quan-

1) Z. anal. Chem. 46. 320 (1907); diese Z. 21, 500 (1908). titativen Bestimmung eignet, konnte ich letzthin in einigen praktischen Fällen feststellen. Dies war die Veranlassung, die genannte Reduktionsmethode bei Abwesenheit von Fluoriden zunı Nachweis von Schwefelverbindungen auf ihre Empfindlichkeit zu prüfen. Hierbei waren unvorhergesehene Schwierigkeiten zu überwinden, die erst dann zutage traten, als bei diesen Versuchen die Empfindlichkeitsgrenze erreicht wurde. Es zeigte sich nämlich, $\operatorname{da} \beta$ von den zu der Reduktion benutzten Materialien eines oder mehrere geringe Mengen Schwefel enthalten mußten. In Frage kamen hierbei: Natriumcarbonat (Natrium-Kaliumcarbonat), das Filtrierpapier und die Heizflüssigkeit, das Ligroin. Die vielen Versuchsreihen, durch welche diese Frage glatt geklärt wurde, mögen übergangen werden. Es gelang nachzuweisen, daB die als chemisch rein gelieferten Präparate wio Natriumcarbonat, Kaliumcarbonat, Natriumbicarbonat frei von S-Verbindungen waren, daß dagegen das benutzte Filtrierpapier (von der Firma Flinsch) in der bekannten Bogenform, ferner das Ligroin schwefelhaltig waren, wenn auch nur in ganz geringen Mengen.

Es kam zunächst darauf an, das Ligroin $\mathrm{zu}$ ersetzen durch eine andere völlig S-freie Flüssigkeit. Hochsiedender Petroläther oder absoluter Alkohol erwiesen sich als unbrauchbar, da sie keine anhaltende Stichflamme lieferten. Im Handelsbenzol lieB sich bequem und in reichlicher Menge Thiophen nachweisen. Nur das sogenannte molekulare Benzol, wie es $\mathrm{zu}$ Molekulargewichtsbestimmungen $\mathrm{z}$. B. von $K$ a h 1 b a u m geliefert wird, wurde als brauchbar erfunden. Benzol übrigens, dem 10\% absol. Alkohol beigemengt worden war, eignete sich nicht dazu, da die Lötrohrlampe zu einer dauernden Stichflamme nicht zu bringen war.

Von den Filtrierpapiersorten sind die sogenannten quantitativen Filter völlig S-frei, ebenso der größere Teil, der von den Firmen Schleicher \& Schüll und Dr. Dreverhoff stammenden abgeteilten glatten Filter. 
Schließlich zeigte es sich noch, daß die Gase des inneren Kegels der Lötrohrlampe allein nicht genügen, um die gesamte Menge Sulfat, sofern es als solches im Untersuchungsobjekte vorhanden ist, zu reduzieren. Es müssen außer der Papierhülse unbedingt noch in genügender Menge $(=1$ runden Filter von ca. $9 \mathrm{~cm}$ Durchmesser) zerschnittenes oder zerzupftes Filtrierpapier zugegen sein, wie ich es in der 1. Mitteilung über diesen Gegenstand bereits angegeben habe.

Die Empfindlichkeit des Nachweises kann man noch erhöhen, wenn man das Untersuchungsobjekt mit Filtrierpapierschnitzeln, ca. $0,1 \mathrm{~g}$ Soda und einigen Grammen einwandsfreiem destillierten Wasser in einer Platinschale zusammenbringt und das Ganze über einer Spirituslampe zur Trockne eindampft, ohne daß Verkohlung der Filtersubstanz eintritt ${ }^{2}$ ). Nach Zusatz von $0,2 \mathrm{~g}$ wasserfreier Soda wird wie weiter unten angegeben verfahren.

Es ist einleuchtend, $\mathrm{da} \beta$ man, um vergleichbare Analysenresultate zu erhalten, die Herrichtung des Sodagemisches durchaus in gleichmäßiger Weise vornehmen muB.

Hat man sich die Gewißheit verschafft, daß die zu benutzenden Materialien völlig S-frei sind, so gestaltet sich nach dem Gesagten der Nachweis folgendermaßen.

Liegt das Untersuchungsobjekt in fester Form vor, so wird es zusammen mit $0,1 \mathrm{~g}$ Natriumcarbonat und der nötigen Menge Filtrierpapierschnitzeln in einer Schale mit einigen Grammen destilliertem Wasser übergossen und das Ganze über einer Spirituslampe zur Trockne eingedampft. Der trocknen Masse werden noch $0,2 \mathrm{~g}$ wasserfreie Soda mittels Pistills zugerieben. Das Gemisch wird nun auf ein Filtrierpapierstück von ca. $5 \mathrm{~cm}$ Seitenlänge gebracht und der Rest aus der Schale mit feuchtem Filtrierpapier herausgewischt und zum übrigen Gemisch gefügt. Durch festes Zusammenwickeln der Papierhülse wird ein Röllchen geformt, an.welches in 3-4facher Windung ein geeigneter Platindraht (an einem Glasstabe befestigt) geschlungen wird ${ }^{3}$ ). Das Sodagemisch des Röllchens wird durch die Flamme der mäßig brennenden Lötrohrlampe zum Schmelzen gebracht. Die Erhitzungsdauer beträgt durchschnittlich 7-8 Min., in manchen Fällen auch 6,9 oder $10 \mathrm{Min}$. Die erkaltete Schmelze enthält nur noch wenige C-Partikelchen, kann übrigens in dieser kompakten Form und trocken längere Zeit aufbewahrt werden, um mit anderen Schmelzprodukten zusammen auf S-Gehalt untersucht werden. Die Schmelze wird in kaltem destillierten Wasser aufgelöst und die Iösung filtriert. Das Filtrat ist in den meisten Fällen farblos, manchmal mit einem Stich ins Gelbliche.

Zum Nachweis von gebildetem Natriumsulfid (durch Geruch meist wahrnehmbar) benutzte ich mit Natronlauge stark alkalisch gemachte Bleiacetatlösung (10 Tropfen $\mathrm{n}$. Bleilösung $+70 \mathrm{~g}$ dest.

2) Bei dieser abgeänderten Versuchsanordnung gelang es mir, in $10 \mathrm{~g}$ einer Probe destillierten Wassers einen S-Gehalt deutlich nachzuweisen.

3) DaB die Finger hierbei frei von Zigarrenrauch usw. sein miissen, braucht wohl kaum gesagt zu werden.
Wasser $+30 \mathrm{ccm}$ 2-n. $\mathrm{NaOH}$ ), da, wie bekannt, eine alkalische Bleilösung empfindlicher ist als eine mit Essigsäure angesäuerte. Da es mir bei diesen Versuchen auf den Nachweis von geringen Mengen S ankam, dienten zur Prüfung der Färbung Reagensgläser aus farblosem Glase mit gerader Bodenfläche. Um die Empfindlichkeit dieses Nachweises zu ernitteln, benutzte ich eine $1 / 1000-n$. Natriumsulfatlösung ( $1 \mathrm{ccm}=0,000096 \mathrm{~g} \mathrm{SO}_{4}$ ). Die Heizflüssigkeit der Lötrohrlampe bestand aus $K$ a h l b a u m schem molekularen Benzol; nur in einem Falle (s. nachstehende Tabelle unter IV) wurde zum Vergleiche Ligroin benutzt. Bei der Untersuchung von Gerste und Malz (s. folgende Tabelle unter III) wurde das Korn unter Zusatz von $0,1 \mathrm{~g}$ Soda und einigen Tropfen Wasser zerrieben; die weitere Behandlung war dieselbe wio oben angegeben.

Auf S. 1260 folgen nun die Versuchsergebnisse, tabellarisch angeordnet.

Wie aus dieser Tabelle (unter $I$ a und b) ersichtlich wird, kann man bei der gegebenen Versuchsanordnung (unter Benutzung von einwandsfreien Materialien) einen Schwefelgehalt von 8/1000 mg S nachweisen. Diese Empfindlichkeit und. die Sicherheit wird von keinem anderen Nachweise übertroffen. Der Schwefelgehalt von Papiersorten (II a, b und c) rührt bekanntlich her von den bei der Verarbeitung des Papiers gemachten Zusätzen wie Alaun, Leim, Gelatine usw. Ich glaube, daß bei Prüfungen von Papiermaterial z. B. auch in gerichtlich-chemischen Fällen diese Reduktionsmethode mit Vorteil angewendet werden kann. Von Interesse ist, daß dio Probe Zigarettenpapier (II d) aus fast völlig reiner Cellulose besteht. Die Versuche unter III b und c wurden größtenteils zu dem Zwecke vorgenommen, zu ermitteln, ob die (übrigens geringe) Verschiedenheit in der Extraktausbeute bei den zwei Malzproben sich durch eine Verschiedenheit im S-Gehalte kundgibt. Dies ist, was die beiden Proben anbetrifft, nicht der Fall. Vielleicht würde man bei einem größeren Unterschiede im Extraktgehalt zu einer klareren Kenntnis kommen. Der Versuch unter IV lehrt, daß der S-Gehalt des Ligroins (bei einer Erhitzungsdauer von $8 \mathrm{Min}$.) nicht so beträchtlich ist, als daß es nicht als billige Heizflüssigkeit in vielen Fällen Verwendung finden könnte.

Zum Schlusse möge noch angegeben werden, daß nach obiger Methode in den off. Fingerhutblättern (von C a e s a r \& L o r e $\mathrm{tz}$ bezogen) rund $0,1 \% \mathrm{~S}$ nachgewiesen wurde; es konnte die interessante Tatsache festgestellt werden, daB diese S-Verbindungen vollständig in den wässerigen Auszug der Blätter (sog. Infusum, bereitet nach der Vorschrift des Deutschen Arzneibuches) übergehen.

Nach den bisher gemachten Angaben eignet sich auf Grund einschlägiger Versuche folgender qualitativer Nachweis von Sulfat neben Fluorid sehr gut für analytische Zwecke.

Das natriumcarbonathaltige Untersuchungsobjekt (ca. 0,2 g) läßt man zusammen mit wenig Wasser von S-freiem Fitrierpapier aufsaugen; auf siedendem Wasserbade wird das Ganze zur Trockne gebracht. Dies hat den Zweck, die Soda möglichst krystallwasserarm zu erhalten. Die vollgesaugten, trocknen Filterstückchen werden hierauf ín ein Papierröllchen von $2-3 \mathrm{~cm}$ Seitenlänge fest ein 


\begin{tabular}{|c|c|c|c|c|c|c|}
\hline \multirow[b]{2}{*}{ I a) } & & & \multicolumn{2}{|c|}{$\begin{array}{l}\text { Erhitzungs. } \\
\text { (dauer in } \\
\text { Minuten) }\end{array}$} & $\begin{array}{l}\text { Farbung nach } \\
\text { Zusatz einiger } \\
\text { Tropfen der } \\
\text { Bleilosung }\end{array}$ & \\
\hline & Sodagemisch & enthaltend & $0,0000144 \mathrm{~g} \mathrm{SO}_{4}=0,0000048$ g S & 7 & stark gelb & \\
\hline b) & $n$ & n & $0,0000091 \mathrm{~g} \mathrm{SO}_{4}=0,00000303 \mathrm{~g} \mathrm{~S}$ & 6 & deutlich gelblich & \\
\hline II a) & $\pi$ & $"$ & $\begin{array}{l}16 \text { qcm bestes Kanzleipapier } \\
\text { (Wasserzeichen: Reichsadler) }\end{array}$ & 8 & $\begin{array}{c}\text { undurchsichtig } \\
\text { schwarzbaun }\end{array}$ & $\begin{array}{l}\text { Furbung be- } \\
\text { stimmt in je } \\
1 \mathrm{~g} \text { des } 21 \mathrm{~g}\end{array}$ \\
\hline b) & $n$ & $n$ & $\begin{array}{l}16 \text { qcm gates Kanzleipapier } \\
\text { (ohne Wasserzeichen) }\end{array}$ & 10 & braungelb & $\begin{array}{l}\text { betragenden } \\
\text { Filtrates der } \\
\text { Schmelze }\end{array}$ \\
\hline c) & $n$ & s & $\begin{array}{l}\text { ca. } 25 \text { qcm gewöhnliches Filtrierpapier } \\
\text { in Bogenform (Firma Fl.). . }\end{array}$ & 9 & braungelb & \\
\hline d) & $n$ & " & $\begin{array}{l}2 \text { Stick schwachgummiertes französ. } \\
\text { Zigarettenpapier „Job“ (Firma } \\
\text { Jean Bardon); gummierter Rand } \\
1 \text { mm breit, Gewicht der beiden }\end{array}$ & & & \\
\hline III a) & $"$ & $n$ & $\begin{array}{l}\text { Papiere }=0,08 \mathrm{~g} \\
1 \text { Gerstenkorn (II. Qual.) }\end{array}$ & $\begin{array}{l}9 \\
71 / 2\end{array}$ & $\begin{array}{l}\text { gelblich } \\
\text { stark gelb }\end{array}$ & \\
\hline b) & $n$ & $n$ & $\begin{array}{l}\text { 2 Malakörner (Wassergehalt der Probe } \\
=6,15 \% \text {, Extraktausbeute im } \\
\text { Feinmehl }=71,25 \% \text { auf Trok- }\end{array}$ & & & \\
\hline c) & $n$ & $r$ & $\begin{array}{l}\left.\text { kensubstanz ber. }=75,91 \% \%^{4}\right) \text {. } \\
2 \text { Malzkörner (Wassergehalt }=4,89^{\circ} \% \text {. }\end{array}$ & $81 / 2$ & braungelb & \\
\hline & & & $\begin{array}{l}\text { Extraktausbeute im Feinmehl } \\
=74,20^{\circ} \text {, auf Trockensub- } \\
\left.\text { stanz berechnet }=78,01^{4}\right) .\end{array}$ & 8 & bräunlichgelb & \\
\hline IV & $n$ & $n$ & $\begin{array}{c}\text { S-freies Filtrierpapier (Heizfiussigkeit: } \\
\text { Ligroin). }\end{array}$ & 8 & gelb & \\
\hline
\end{tabular}

4) Die Malzprobe nebst deren Bestimmungen an Extraktausbeute usw. verdanke ich dem Entgegenkommen der Görlitzer Aktienbrauerei. - Näheres über die Bestimmung des Extraktgehaltes findet man z. B. in R öttg e r s „Kurzes Lehrbuch der Nahrungsmittelchemie 1903, 398.

gewickelt und mit einem Platindraht (am Glasstabe befestigt) umwunden. Das Röllchen läßt man in einer Spirituslampe oberflächlich verkohlen und richtet auf dasselbe mittels eines Lötrohres die Stichflamme des Spiritusbrenners. Bei ruhigem und gleichmäßigem Blasen mit dem Lötrohre ist die Reduktion in 3 Minuten beendet. Man legt nun in der bekannten Weise die mit Wasser angefeuchtete Schmelze auf ein blankes Silberstick, oder man benutzt das Filtrat der Schmelze zur Prüfung mit Bleilösung. Ich glaube, daß diese Form des qualitativen Nachweises von Sulfat neben Fluorid an Einfachheit nicht viel $2 u$ wünschen übrig läßt.

Kritisches zu Ehronfelds Arboit: "Zurquantitativen Scheidung von Sulfaten und Fluoridench).

Durch meine Untersuchungen über den Nachweis von Schwefelsäure neben Flußsäure ${ }^{6}$ ) veranla Bt, veröffentlichte voriges Jahr $\mathrm{E} h \mathrm{r}$ enfold zusammen mit $A$. In $\mathbf{r}$ a eine andere analoge Methode, die darauf beruht, die Reduktion von Sulfat zu Sulfid durch Erhitzen mit Zinkstaub vorzunehmen und den durch verd. Schwefelsäure in Freiheit gesetzten Sohwefelwasserstoff qualitativ wie üblich nachzuweisen, während quantitativ derselbe jodometrisch bestimmt wird. Nach Angaben von Ehrenfeld besitzt dieser Nachweis den Vorzug, bequemer und genauer zu sein als der

5) Chem.-Ztg. 1909, 375 .

6) $1 . \mathrm{c}$. meinige, da der ungünstige Einfluß von Fluorid auf Sulfat bei der Reduktion durch einen Zusatz von Kieselsäure und Tonerde auggeschaltet wird. Was nun die qualitative Seite des $\mathrm{E}$ h r e n f e ld schen Nachweises betrifft, so ist zu bemerken, daß Zinkstaub meßbare Mengen von Schwefelverbindungen enthält, die mit verd. Schwefelsäure (15 bis $20 \%) \mathrm{H}_{2} \mathrm{~S}$ entwickeln. Dieser Gehalt an Schwefelverbindungen macht den qualitativen Nachweis Ehrenfelds bis zu einem gewissen Grade illusorisch, falls nicht an Stelle von Zinkstaub gepulvertes Zink „Kahlbaum“ benutzt wird, das als S-frei angesehen werden kann. [A. 104.]

\section{Nachweis von Cruciferenölen in Ö1- gemischen.}

(Mitteilung aus dem Kgl. Materialprafungsamt.)

Von D. Holde und J. Marcosson.

(Eingeg. 4./5. 1910.)

Für Kennzeichnung fetter Öle kommen zurzeit hauptsächlich neben wenigen qualitativen Reaktionen, die zum Teil auch nur bedingt zuverlässig sind, die sog. ,quantitativen Reaktionen“, wie Jodzahl, Säurezahl, Verseifungszahl, R e i c h e r t Me iBlsche Zahl und Acetylzahl in Frage. Nur in wenigen Fällen ist es bisher gelungen, 